\title{
Cambio del modelo metodológico en la asignatura Laboratorio de Farmacia
}

\author{
María Jesús Cejudo BAStANTE \\ Universidad de Sevilla \\ Facultad de Farmacia \\ Dpto. Nutrición y Bromatología, \\ Toxicología y Medicina Legal \\ mjcejudo@us.es \\ ORCID: https://orcid.org/0000-0003-0197-2429 \\ D.O.I.: http://dx.doi.org/10.12795/JDU.2018.i01.07 \\ Pp.: $132-145$
}

\section{Resumen}

El objetivo de esta innovación fue favorecer el carácter crítico en las prácticas mediante un cambio en el modelo metodológico, evitando su realización de manera automática sin plantearse su aplicabilidad. El ciclo de mejora docente se llevó a cabo en las prácticas de la asignatura Laboratorio de Farmacia, impartida en 40 curso del Grado de Farmacia de la Universidad de Sevilla. Una vez expuestos los objetivos de la práctica, los alumnos realizaron junto al profesor un mapa de contenidos de la misma, especificando qué se va llevar a cabo, cómo, porqué y para qué. Los resultados de la encuesta revelaron que aproximadamente el $\mathbf{9 0 \%}$ de los alumnos valoraron la actividad como "buena" y "bastante buena", confirmando que la nueva metodología fue satisfactoria para el alumnado. Este hecho se vio reflejado 
satisfactoriamente en las notas finales, con un porcentaje de aprobados en torno al $90 \%$.

Palabras clave: Laboratorio de Farmacia, Grado en Farmacia, docencia universitaria, experimentación docente universitaria, cambio metodológico.

\section{Breve descripción del contexto}

El Grado de Farmacia de la Universidad de Sevilla consta de 4 años (240 ECTS) y está estructurado en asignaturas con carácter obligatorio, optativo, prácticas tuteladas y el trabajo Fin de Grado (Decanato de la Facultad de Farmacia de Sevilla, 2017). Esta Facultad cuenta con aproximadamente 335 profesores con encargo docente agrupados en 22 departamentos, con un total de 2258 alumnos. Laboratorio de Farmacia, asignatura donde se realiza la mejora docente, es de carácter obligatorio del cuarto curso de dicha titulación, impartida durante el segundo cuatrimestre en la Facultad de Farmacia.

Desde el curso 2012-2013 he participado en proyectos de innovación y mejora docente de la Universidad de Sevilla, por lo que inicialmente cuento con cierta experiencia en la mejora de la docencia. En estos cursos, he realizado ciclos de mejora en seminarios, con unos resultados bastante positivos. Una vez tomada conciencia de la importancia de un cambio de metodología, he aplicado el ciclo de mejora en la parte práctica. Por motivos de una estancia de investigación, lo he aplicado a otra asignatura de la misma área, cuyos contenidos son similares a la asignatura donde normalmente he venido realizando los ciclos de mejora, por lo que si los resultados obtenidos son adecuados, la implantaré en el siguiente curso académico para esa asignatura.

La intervención se realizará en la parte de prácticas de la asignatura Laboratorio de Farmacia del grado de Farmacia (módulo de Nutrición y Bromatología). En concreto, se 
Llevará a cabo en cuatro grupos de clase impartidos en sesiones de mañana y tarde. La innovación se realizará en la misma aula de impartición de las clases, no siendo necesaria ninguna sala ni recurso adicional. La asignatura Laboratorio de Farmacia tiene asignados 6 créditos ECTS y la imparten varios departamentos, entre los que se encuentra el Departamento de Farmacología (2 ECTS), el Departamento de Farmacia y tecnología farmacéutica (2.5 ECTS), y el Departamento de Nutrición y Bromatología, Toxicología y Medicina Legal (1.5 ECTS), departamento al que pertenezco.

\section{Diseño previo del ciclo de mejora docente}

\section{Mapa de contenidos.}

Los objetivos de este módulo de la asignatura son saber usar las técnicas y métodos analíticos fundamentales para la investigación bromatológica en matrices como alimentos (figura 1).

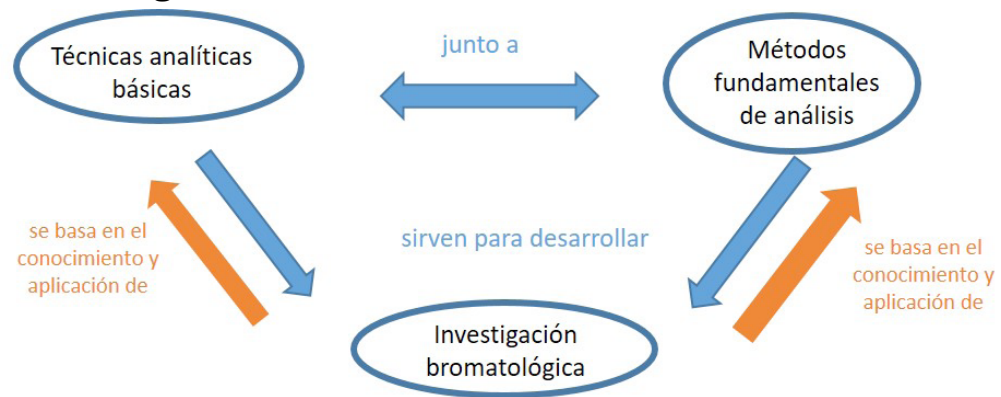

Figura 1. Mapa de contenidos general de la asignatura Laboratorio de Farmacia.

Muchos alumnos se cuestionan la aplicabilidad de las prácticas a realizar y les cuesta conectarlas con los contenidos teóricos impartidos en clase. Por ello, es necesario que el profesor piense de manera esquemática en realizar un mapa de contenidos adecuado y claro para mostrarlo 
así durante el desarrollo de las prácticas. En base a esto, se realizará el ciclo de mejora en el modelo metodológico tratando de que los alumnos construyan su mapa de contenidos sobre la práctica que se va a realizar.

\section{Modelos metodológicos y}

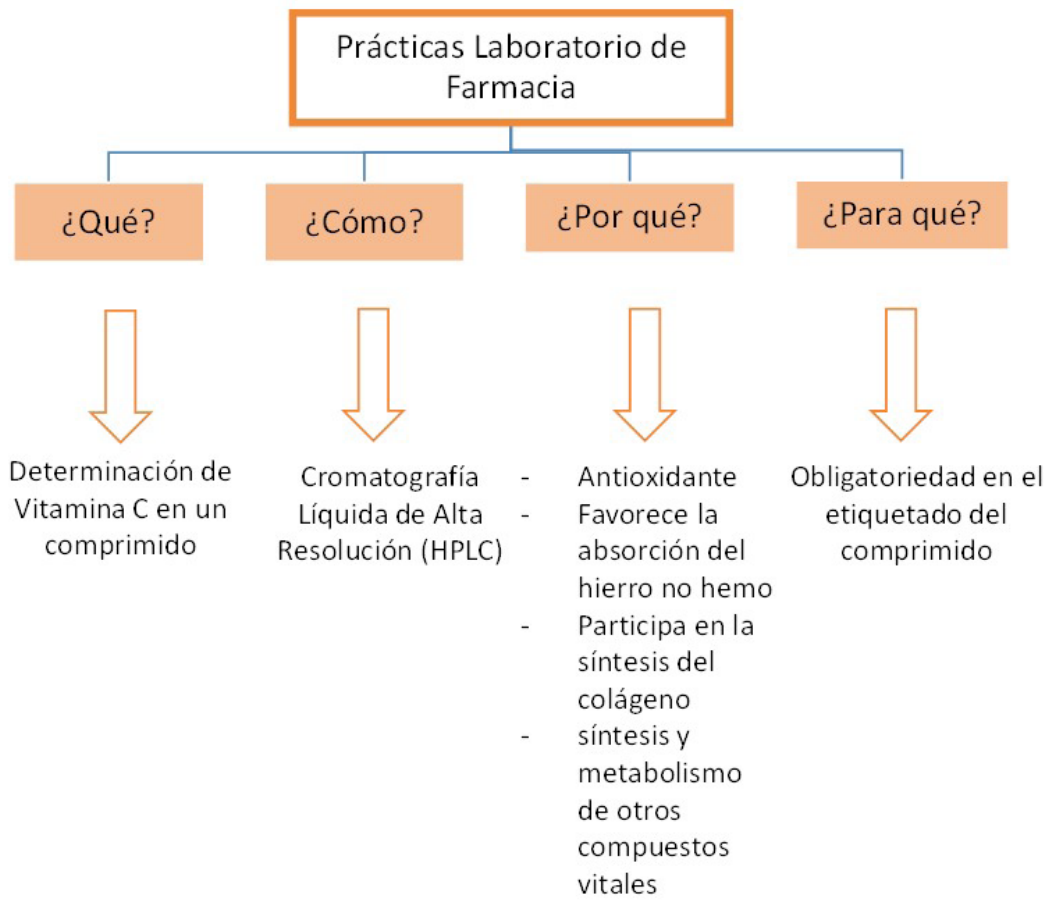

Figura 3. Mapa de contenidos de la práctica de análisis de vitamina C en un comprimido llevado a cabo en la asignatura Laboratorio de Farmacia.

\section{secuencia de actividades.}

Los contenidos de la asignatura Laboratorio de Farmacia se impartirán durante tres sesiones a la semana de tres horas cada día durante el segundo cuatrimestre. El ciclo de mejora se aplicará en todas las sesiones de cada grupo de prácticas. Primeramente se expone el modelo metodológico habitual o convencional y, posteriormente, el modelo 
metodológico posible tras realizar la innovación o mejora (De Miguel, 2005).

\section{Modelo metodológico habitual}

El modelo metodológico habitualmente aplicado consta de cuatro partes: introducción, práctica, conclusiones y limpieza de material. Se comienza diciendo a los alumnos que, puesto que el protocolo de prácticas les ha sido entregado previamente por enseñanza virtual, deben conocer el contenido de las mismas y cómo realizarlas. Así, el profesor desarrolla una explicación de la práctica y los alumnos comienzan a realizarla. Una vez terminada, el profesor pregunta cuáles son las conclusiones de la práctica, intentando crear un debate que pocas veces se consigue. Según lo descrito, la modelización de una clase habitual de prácticas tendría un modelo como el que se muestra en la figura 2. 


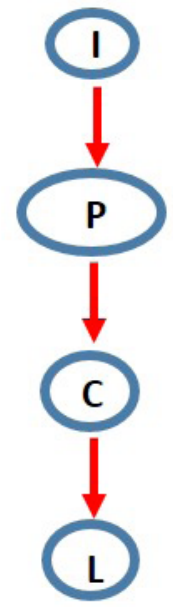

Figura 2. Modelo metodológico seguido en la docencia de las clases de Laboratorio de Farmacia. I, Introducción (explicación teórica del fundamento de la práctica); P, Prácticas (Resolución de las prácticas); C, Conclusiones; L, Limpieza del material de laboratorio.

De esta forma, por experiencia propia en años anteriores, a pesar de que el protocolo de prácticas se les proporciona previamente a través de enseñanza virtual como se ha comentado anteriormente, prácticamente ningún alumno las lee previamente. Además, percibo que los alumnos se diluyen durante la parte de explicación teórica, y están más interesados en empezar cuanto antes la práctica para acabar lo antes posible. Como consecuencia, estos hechos provocan que realicen la práctica de manera automática, sin pensar en lo que están haciendo, porqué lo hacen y porqué de esa manera y, mucho menos, para qué sirve.

En todas las fases se intenta favorecer la interacción entre profesor y alumno, aumentar su participación y atender a sus ideas e intereses (Bain, 2007), de acuerdo con los principios didácticos expuestos anteriormente.

Jornadas de Formación e Innovación Docente del Profesorado | № 1 (2018) Esta obra se distribuye con la licencia Creative Commons 
La secuencia de actividades a cada una y el tiempo dedicado en cada una de las sesiones es la que a continuación se indica:

1. Breve introducción de la temática de la práctica (5 minutos).

2. Desarrollo de la práctica por los alumnos (2.5 horas)

3. Exposición de conclusiones. Trabajo personal de los alumnos y apoyo del profesor (10 minutos).

4. Limpieza del material (15 minutos).

Los porcentajes aproximados de cada una de las partes son $5,5,85$ y $10 \%$, respectivamente, siendo consecuente con el modelo metodológico.

Aprovechando el carácter obligatorio de la asignatura (asignatura práctica), por todo lo expuesto anteriormente, los alumnos realizan las prácticas de manera automática sin plantearse la aplicabilidad de las mismas. Por lo tanto, resulta interesante favorecer en los alumnos el carácter crítico en las prácticas, para lo cual se plantea el ciclo de mejora.

Modelo metodológico posible en el ciclo de mejora docente y secuencia de actividades

Las preguntas clave fueron “¿Para qué sirve la práctica que voy a realizar?” y “¿Para qué sirve poder realizar correctamente este tipo de análisis y qué importancia tiene la información que nos puede dar?". La innovación consistió en comentar a los alumnos solo el título de la práctica y las preguntas clave y, a continuación, iniciar una exposición sobre su importancia (objetivo), cómo lo harían y qué aparatos utilizarían y, posteriormente, la aplicabilidad de los resultados que obtendrían. El objetivo es la toma de conciencia y expresión por parte de los estudiantes de sus ideas respecto al problema (ideas iniciales de los estudiantes). De esta forma, se evitaría la resolución automática de las prácticas sin cuestionarse el planteamiento, hecho que ocurre en prácticamente todas las prácticas que he realizado en mis años de docencia. El profesor pretende 
que los alumnos sean los que planteen el objetivo y la resolución de las prácticas y no sea tipo clase magistral, de manera que el profesor solo vaya orientando a los alumnos en cada práctica. Además, una vez expuestos los objetivos de la práctica, se realizó junto al profesor un mapa de contenidos de la misma, especificando qué se va llevar a cabo, cómo, porqué y para qué (figura 3).

Así, el modelo metodológico habitual se modificó, sobre todo en la primera y tercera parte, ya que se empleó un tiempo superior al empleado anteriormente, pero necesario para que los alumnos tomen conciencia desde el principio del objetivo y la aplicabilidad de cada práctica a realizar. Posteriormente a la realización de la práctica, se expusieron las conclusiones, en las que se manifestaron las ideas finales y se compararon con las iniciales mediante el debate. Finalmente, los alumnos procedieron a la limpieza del material empleado en la elaboración de la práctica.

La secuencia de actividades y el tiempo dedicado a cada una de ellas fueron los siguientes en cada una de las sesiones (figura 4):

1. Exposición de la pregunta clave, en el que se expusieron las ideas iniciales de los estudiantes y se elaboró un mapa de contenidos (30 minutos)

2. Desarrollo de la práctica por los alumnos (2 horas)

3. Exposición de conclusiones en las que se expusieron las ideas finales. Debate comparativo con las ideas iniciales y sobre lo aprendido en el proceso (15 minutos)

4. Limpieza del material (15 minutos)

\section{MODELO METODOLÓGICO MODIFICADO}

\section{PC-II}

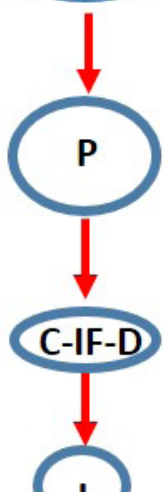


Los porcentajes aproximados de cada una de las partes fueron 10, 70, 10 y $10 \%$, respectivamente, siendo consecuente con el modelo metodológico.

El reajuste de tiempos por actividad es fácilmente realizable pues el tiempo que se destina a la nueva actividad no es demasiado elevado.

Cuestionarios de evaluación

La consecución de los objetivos de la nueva metodología empleada se quiso constatar mediante un cuestionario de opinión que realizarán al término de la práctica (Anexo 1) (Porlán, 2017). El mismo consta de cuatro preguntas cerradas tales como: 1, "valore su satisfacción con la metodología aplicada durante la práctica"; 2, “Cree necesaria la aplicación de esta metodología en el resto de prácticas del grado?"; 3, "¿Piensa que ha entendido mucho mejor la práctica con la metodología aplicada?" y 4, "En términos generales, mi valoración sobre la metodología impartida es:", con cinco opciones del modo "nada", "poco", "indiferente", "buena" y "bastante" e indicando la razón de dicha calificación.

El objetivo del cuestinariovalorar si la metodología aplicada había sido satisfactoria en relación a la manera en la que normalmente suelen impartirse las prácticas. De hecho, en el cuestionario se les dejará un espacio para que expresen su opinión comparativa.

\section{Aplicación del ciclo de mejora docente}

\section{Relato resumido de las sesiones.}

Según la secuencia de actividades expuesta en el punto 3.2, se les dijo a los alumnos que no se leyeran la práctica, lo cual a muchos les llamó la atención. A continuación, el profesor entabló un diálogo mediante las preguntas clave expuestas en la figura 3, siendo esta figura el 
mapa de contenidos formando en base al diálogo con los alumnos. Eran alumnos de cuarto curso, por lo que había alumnos que respondían a las preguntas adecuadamente, aunque en la mayoría de los casos fue el profesor el que les iba guiando y dando muchas de las soluciones, dejándoles siempre pensar y razonar antes. Una vez expuesta toda la información y construido el mapa de contenidos, se inició el desarrollo de la práctica por los alumnos, de manera más autónoma que mediante el modelo metodológico habitual.

Una de las mayores dificultades que se presentaron fue el cálculo del contenido de vitamina C, teniendo en cuenta dos perspectivas: los alumnos tienen dificultad en tener en cuenta las diluciones realizadas y tienen una gran inseguridad a la hora de afirmar que el dato que están dando es correcto, siendo ambos conceptos y tareas que se deberían dominar al tratarse de una asignatura de cuarto curso. Posteriormente, se realiza una limpieza de material y se da por terminada la práctica. Otra dificultad es que, en ocasiones, los grupos de prácticas son muy numerosos y si la práctica no se entiende bien desde el principio, hace que se retrase el desarrollo de la misma. En esos casos, aunque siempre manteniendo la premisa de hacer razonar a los alumnos, el profesor estuvo más pendiente de los grupos con más dificultades.

\section{Evaluación del aprendizaje de los estudiantes.}

Antes de empezar la práctica y durante la exposición de las ideas iniciales, algunos alumnos no tenían demasiada idea del objetivo ni de la metodología necesaria para calcular el contenido de vitamina $\mathrm{C}$ de un comprimido. Tras la explicación y posterior exposición del mapa de contenidos elaborado entre los alumnos y el profesor, los alumnos fueron capaces de exponer la aplicabilidad de la práctica y el procedimiento a seguir. Por lo tanto, se puede afirmar 
que el objetivo de la nueva metodología se consiguió en gran medida.

En cuanto a la evaluación del alumnado, aunque la participación en clase se evaluó positivamente, el resultado del examen final de prácticas reveló un porcentaje elevado de aprobados (90\%), de los cuales el $40 \%$ obtuvieron notas entre 9 y 10, el 30\% entre 7 y 9, lo que está estrechamente relacionado con el buen entendimiento de la metodología de aprendizaje empleada.

\section{Evaluación del ciclo de mejora docente}

Entre los 32 alumnos encuestados, los resultados de la encuesta revelaron que aproximadamente el $90 \%$ respondieron a las cuatro preguntas con una respuesta de "buena" y "bastante", lo que confirma que la nueva metodología fue satisfactoria para el alumnado, ya que entendieron en mayor medida la práctica realizada y que les interesa su implantación en el resto de prácticas del grado de farmacia. Entre los comentarios positivos se encuentran "nos ayuda a razonar lo que estamos haciendo y no solo a realizar lo que dice el protocolo", "porque ha explicado todo paso por paso y asegurándose de nuestro entendimiento y razonamiento", "muchas veces nos limitamos a seguir el guión de prácticas sin saber realmente", "con esta metodología el alumno razona y entiende mejor la práctica, lo que supone tenerlo todo más claro a la hora de realizarla" y "porque cuando no se explica no nos enteramos de nada, cometemos más errores y después en el examen pasa factura". Además, a pesar de haber valorado la nueva metodología como "buena" o "bastante", hubo dos alumnos que expresaron diferentes opiniones al resto, tales como "creo que hay que explicar la práctica en su justa medida, es decir, decir lo importante. Si se explica la práctica en una hora aburre a los alumnos", "depende de la práctica; si es de dificultad alta se agradece que se explique y detalle el desarrollo de la misma" y "cuando hay 
un tiempo limitado, si tenemos que llegar nosotros solos a unas conclusiones sin guía, el tiempo es mucho mayor, superándose el número de créditos". Por último, hubo un alumno que contestó con "poco" o "indiferente", alegando que "prefiero entender por mí mismo las posibles dudas, para así acostumbrarme cuando llegue a un nuevo trabajo y con ello ser más resolutivo" o "en las prácticas de primeros años viene bien explicar, pero a medida que avanzamos debemos ser más resolutivos".

De manera general, según la experiencia de este curso académico y viendo los resultados obtenidos en el cuestionario, incorporaré este modelo metodológico a mi práctica docente habitual en cada clase, para así afianzar conceptos y que ellos piensen la aplicabilidad de los mismos. Además, para el profesor ha sido mucho mejor ya que el hecho de que la práctica se entienda antes de realizarla hace que se eviten preguntas/dudas a los largo del desarrollo de la misma y que los alumnos sean mucho más autónomos.

\section{Cuestiones a mantener y cambios a introducir.}

Tras la aplicación del ciclo de mejora en el modelo metodológico de la sesión práctica de la asignatura Laboratorio de Farmacia del Grado de Farmacia, y tras analizar los resultados del cuestionario de evaluación de los alumnos, pienso introducir el modelo metodológico aplicado. Sin embargo, pienso que es necesario que los alumnos piensen por ellos mismos, por lo que la primera parte del modelo metodológico aplicado la voy a introducir en la práctica, pero no así guiarles tanto en el cálculo matemático de los resultados, puesto que en cuarto curso deberían realizarlo sin problemas. 


\section{Aspectos de la experiencia que se pretenden incorporar a toda la práctica docente habitual.}

Tras la buena aceptación que ha tenido el nuevo modelo metodológico, pienso incorporar el mismo a toda la práctica docente habitual, en términos de realizar una buena explicación guiada, no magistral, de los contenidos que se van a enseñar, cómo, por qué y con qué finalidad, y en la medida de lo posible, conectarlos con los contenidos teóricos con el fin de que los alumnos sean conscientes de la aplicabilidad de la práctica a realizar.

\section{Principios didácticos argumentados.}

1. Incrementar la comprensión y asimilación de los contenidos.

2. Establecer relaciones entre contenidos, elaborando modelos más completos y útiles.

3. Propiciar el sentido crítico de los alumnos enfatizando en la aplicabilidad de lo que se está haciendo.

\section{Referencias bibliográficas}

Bain, K. (2007). Lo que hacen los mejores profesores universitarios. Universidad de Valencia: Publicaciones de la Universidad de Valencia.

Decanato de la Facultad de Farmacia de Sevilla (2017). Guía de la Facultad de Farmacia curso 2017/2018. Recuperado de http://www.farmacia.us.es/guia.

De Miguel, M. (2005). Metodologías de enseñanza para el desarrollo de competencias. Orientaciones para el profesorado universitario ante el Espacio Europeo de Educación Superior. Madrid: Alianza.

Porlán, R. (2017). Enseñanza universitaria. Cómo mejorarla. Universidad de Sevilla: Ediciones Morata, S. L. 


\section{ANEXO 1 \\ CUESTIONARIO DE EVALUACIÓN DE LA INNOVACIÓN DOCENTE}

\section{Grupo:} Fecha:

Este cuestionario contiene una serie de preguntas relativas a la metodología docente impartida en la asignatura Laboratorio de Farmacia, del Grado de Farmacia. La metodología impartida por el profesor se enmarca dentro del programa de formación e innovación docente del profesorado, dentro de la Red para la formación e innovación docente (REDIF).

Por favor, lea atentamente cada pregunta y responda según su criterio. Sea lo más objetivo posible. Su respuesta será un indicador de gran utilidad sobre aquellos aspectos y conductas docentes de innovación a implementar en la asignatura.

\begin{tabular}{|c|c|c|c|c|c|}
\hline & Nada & Poco & Indiferente & Buena & Bastante \\
\hline $\begin{array}{l}\text { 1. Valore su satisfacción con la met } \\
\text { dología aplicada durante la práctica }\end{array}$ & & & & & \\
\hline Indique la razón de esa calificación & & & & & \\
\hline $\begin{array}{l}\text { 2. ¿Cree necesaria la aplicación } \\
\text { de esta metodología en el resto de } \\
\text { prácticas del grado? }\end{array}$ & & & & & \\
\hline Indique la razón de esa calificación & & & & & \\
\hline $\begin{array}{l}\text { 3. ¿Piensa que ha entendido mucho } \\
\text { mejor la práctica con la metodologí } \\
\text { aplicada? }\end{array}$ & & & & & \\
\hline Indique la razón de esa calificación & & & & & \\
\hline $\begin{array}{l}\text { 4. En términos generales, mi valo- } \\
\text { ración sobre la metodología impar- } \\
\text { tida es: }\end{array}$ & & & & & \\
\hline Observaciones: & & & & & \\
\hline
\end{tabular}

MUCHAS GRACIAS.

Jornadas de Formación e Innovación Docente del Profesorado I № 1 (2018) Esta obra se distribuye con la licencia Creative Commons Reconocimiento-NoComercial-SinObraDerivada Internacional (CC BY-NC-ND 4.0.) 